\title{
Superhydrophobicity in hierarchically nanostructured metal surfaces
}

\author{
Pushan Ayyub \\ Department of Condensed Matter Physics \& Materials Science, Tata Institute of \\ Fundamental Research, Homi Bhabha Road, Mumbai 400005, INDIA \\ Email: pushan@tifr.res.in
}

Many naturally occurring surfaces (duck feathers, lizards' feet, lotus leaf) are superhydrophobic, i.e., the contact angle for water is very high $\left(>140^{\circ}\right)$. Such naturally occurring surfaces may show interesting differences. Both rose petals and lotus leaves are superhydrophobic, but while water drops quickly roll off a lotus leaf, they adhere strongly to a rose petal. Whether the hydrophobic surface is 'slippery' (lotus leaf) or 'sticky' (rose petal) cannot be characterized by just the contact angle, and requires an additional parameter. The coexistence of these apparently contradictory properties - strong adhesion and super-hydrophobicity - is usually ascribed to the complexity of the surface microstructure. In this talk, we discuss how the hierarchical nature of the surface - structured at both the micro and the nano scale - can give rise to a whole range of surface properties.

We show that the clustering of a parallel array of metal nanorods exhibits a type of emergent behavior [1], which can be utilized to produce a two-level hierarchical structure with superhydrophobic surface [2] that is required for tuning the hysteresis in the contact angle. The contact angle hysteresis is the parameter that controls whether the surface is slippery or sticky. We can thereby obtain either the lotus leaf effect or the rose petal effect by slight modifications of essentially the same system [3]. The cluster morphology of the nanorod arrays - electrochemically grown within porous alumina templates - is determined by the template geometry and drying conditions. Such a clustered metal nanorod array provides a simple model system for studying the coexistence and complex interplay of hydrophobicity and adhesion. It also provides some degree of control over these mutually contradictory properties.
We have also shown that the clustering of metal nanorod arrays can yield other interesting results (Figure 1). A suitable optimization of the cluster geometry leads to large enhancements in the field-induced electron emission and laser-induced X-ray emission.
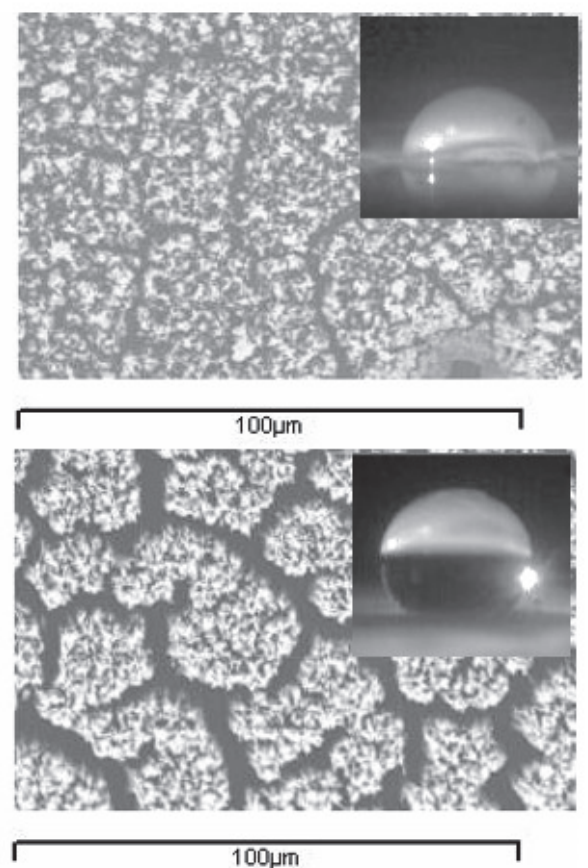

Figure 1: Surface view scanning electron micrographs of an unclustered (top) and a heavily clustered (bottom) metal nanorod array. The shape of the water droplets (inset) indicate that these surfaces are, respectively, hydrophilic and superhydrophobic

\section{References}

1. P Ayyub: J. Cluster Science 20 (2009) 429-451

2. P Bhattacharya, S Gohil, J Mazher, S Ghosh, $P$ Ayyub, Nanotechnology 19 (2008) 075709

3. I Chakraborty, N Singh, S Gohil, S Ghosh, P Ayyub: Soft Matter 9 (2013) 11513-11520 